\title{
Using Blood Vessels Location Information in Optic Disk Segmentation
}

\author{
A.S. Semashko ${ }^{1}$, A.S. Krylov ${ }^{1}$, and A.S. Rodin ${ }^{2}$ \\ 1 Laboratory of Mathematical Methods of Image Processing, \\ Faculty of Computational Mathematics and Cybernetics, \\ Lomonosov Moscow State University \\ http://imaging.cs.msu.ru \\ 2 Ophthalmology Chair, Faculty of Fundamental Medicine, \\ Lomonosov Moscow State University
}

\begin{abstract}
In this paper we present an approach to achieve high accuracy of optic disk segmentation using information on the location of blood vessels (vessel map). Morphological preprocessing is employed to remove the vessels from the image and to compute the vessel map. Vessel map is combined with edge map to obtain robust initial approximation of OD boundary using circular Hough transform. We use this approximation to build 2D weight function for the edge map, which is then used in the active contour model. We introduce an additional step to perform correction of the contour; in this step, the active contour model includes pressure forces and soft elliptical constraint. Vessel map is used in calculation of the ellipse parameters and pressure values. The method was tested on 1240 publicly available retinal images, and manual labeling of the disk boundary by medical experts was used to assess its accuracy and compare it with other optic disk segmentation methods.
\end{abstract}

\section{Introduction}

Optic disk (OD) is the region where optic nerve and blood vessels pass through the sclera. In automated retinal image analysis, locating the optic disk boundary is one of the most important tasks. Changes in optic disk appearance are used by ophthalmologists to assess the progression of diseases and treatment. Particularly, size of the optic disk cup is crucial in the study of patients with glaucoma. Other than being an indicator for various pathologies, optic disk is used as a reference to measure distances and detect other anatomical parts of the retina.

There are various methods for finding the exact shape of the disk. A thorough review is available in [17], so in our paper we will focus on the most referenced and/or close to our work methods only. Lalonde et al. [8] proposed an OD localization scheme using Hausdorff based template matching and pyramidal decomposition. This method assumes a circular model to approximate OD region for which radius parameter was estimated from the localized region. $\mathrm{Li}$ et al. [9] used an active shape model with some modifications that improve the 
method robustness in optic disk segmentation. Lowell et al. [10] used an elliptical deformable model with multi-stage boundary detection: the more salient temporal-side edge is detected first. In [4] a method based on blob detection is presented that is designed to be insensitive to image quality and not dependent on the image registration method.

Many OD segmentation methods employ specific models of disk shape, which makes them unable to precisely segment optic disks of irregular shapes. A number of freeform active contour based methods was presented recently to tackle irregular optic disk shapes as well. Mendels et al. [11] proposed to use gradient vector flow snake with a preprocessing step that removes blood vessels. Osareh et al. [13] improve the previous work by using a template matching scheme for automatic snake initialization and performing preprocessing in three-dimensional CIELab color space. Xu et al. [19] developed a freeform snake approach and report performance improvement over their previous results [9], however the segmentation accuracy is highly dependent on contour initialization. Tang et al. [16] proposed a Chan and Vese (C-V) model [2] based method, but their model incorporate an elliptical shape restraint. Joshi et al. [6] presented a C-V based method which does not impose any shape constraint to the underlying model. Instead, the model differentiates the OD region from the similar characteristic regions around it by integrating information from multiple image feature channels: the red color image channel and two texture-space channels.

Most freeform model-based methods include a preprocessing step that removes blood vessels, because their edges would affect the model evolution, severely distracting the result. In [6] blood vessels are segmented first, then the values at vessel points are somehow interpolated using nearby non-vessel regions. The approach employed in [11][13] uses, on the contrary, a simple but powerful vessel removal technique based on mathematical morphology, which works fine just because of the nature of the retinal image.

Optic disk segmentation methods based on some kind of freeform model that requires an image without blood vessels to perform well, share one common drawback: optic disk boundary in the places where it is crossed by blood vessels is not treated specifically. Such behavior implies that the vessel removal technique should recover the true optic disk shape, which is typically not the case. For example, the approach used in [11][13] often results in small concavities in the optic disk boundary in the places where it is crossed by blood vessels after the preprocessing.

In this paper we present a method based on [13]. In the preprocessing step we detect image areas occupied by blood vessels. This information is used later, both in the preprocessing and correction steps, resulting in significantly higher segmentation accuracy in most cases.

Notes. 1. The paper focuses on the segmentation and does not cover optic disk localization problem. 2. After the disk location is determined, we use only a part of the original image containing the disk to increase the performance. All operations described in this paper use only the cropped image. 


\section{Preprocessing}

Mendels et al. [11] proposed to use grey-level mathematical morphology for removing blood vessels. Osareh et al. [13] carried out a series of experiments comparing the results of morphological closing operation in several color spaces, namely: grey-level, lightness channel L of HLS space, full HLS space, LCh space and Lab space. Between all the color spaces tested, the Lab space allows to get the most homogeneous region and preserves optic disk edges better.

Application of morphological operations in multidimensional color spaces requires a rule for comparing different color values. Osareh et al. used a simple definition of maximum and minimum in Lab space to perform morphological closing: to compare two color values, the Euclidean distance from the color space origin $(0,0,0)$ is computed for both colors and the resulting values are compared. This strategy works fine for optic disk images, though it might not perform well in other applications.

The difference between original image and the result of morphological closing $I_{c}$ is used to calculate vessel map $D$. Each value in this map can be thought of as the Bayesian probability of the corresponding pixel being a vessel pixel. In rare cases a better segmentation may be achieved by applying morphological dilation with a small (1-2 pixels radius) disk-shaped structuring element to the vessel map. An example of a morphological closing result and the corresponding vessel map is shown in Fig. 1 (b, c).

(a)

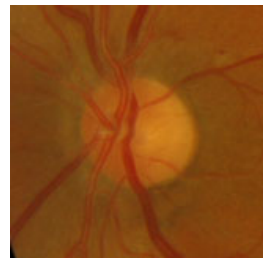

(d)

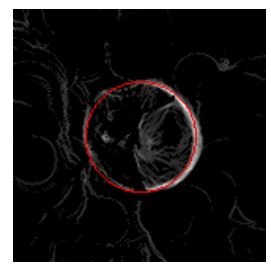

(b)
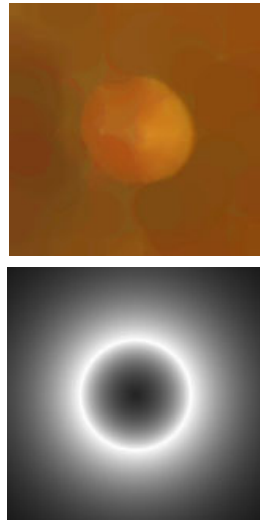

(c)
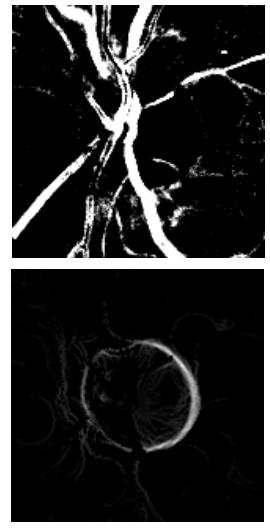

Fig. 1. Sample intermediate results of preprocessing stage. a) Original sub-image; b) result of blood vessels removal $I_{c} ; \mathrm{c}$ ) vessel map $D ; \mathrm{d}$ ) preliminary edge map $M_{\text {pre }}$, and the circle obtained using Hough transform (shown in red); e) edge map scaling coefficient; f) final edge map $M$.

The vessel map and the lightness channel $L_{c}$ in $I_{c}$ are used to produce a preliminary edge map as follows: $M_{\text {pre }}(P)=\left|\nabla L_{c}(P)\right| \cdot(1-D(P)) / \max \left|\nabla L_{c}\right|$. The Hough transform for circles is applied to the preliminary edge map to obtain 
a circular approximation of the optic disk boundary (see Fig. 1, d). This circle is used to construct an initial contour for the active contour model, and also to generate the final edge map $M$. The preliminary edge map typically contains a lot of edges that do not belong to the OD boundary. There may be some pathologies in close vicinity of the disk, and the OD itself often have highly inhomogeneous lightness, which results in strong edges inside the disk. These unwanted edges, though located at some distance of the initializer, may affect the active contour because of the nature of the GVF external force field that used in our work. To suppress them, we multiply each value in the preliminary edge map with a coefficient that is derived from the distance between the corresponding pixel and the circular approximation (see Fig. 1, e).

The resulting edge map $M$ is used to compute the GVF external force field for the active contour model.

\section{Active Contour Models}

Active contour model, specifically the so-called gradient vector flow (GVF) snake [18], is used to localize the optic boundary, and the same model with pressure forces added to it is used in the correction process. Active contour is a parametric curve $\mathbf{v}(s)=(x(s), y(s))^{\mathrm{T}}(s \in[0,1])$ that iteratively moves due to influence of internal and external (image and/or constraint) forces acting upon it. The original snake model [7] is formulated as an energy minimization problem. The basic form (that does not include external constraints) of the energy functional is as follows:

$$
E[\boldsymbol{v}(s)]=\int_{0}^{1}\left(\alpha(s)\left|\boldsymbol{v}(s)_{s}^{\prime}(s)\right|^{2}+\beta(s)\left|\boldsymbol{v}(s)_{s s}^{\prime \prime}(s)\right|^{2}+E_{\mathrm{ext}}(\boldsymbol{v}(s))\right) d s,
$$

where $E_{\text {ext }}$ is determined by the edge map. For example, if edge strength is defined as squared magnitude of the intensity image $I$ gradient, then image energy at point $P$ is $E_{\text {ext }}(P)=-|\nabla I(P)|^{2}$. In discrete formulation, Euler equations for the curve that minimizes Functional (1) are

$$
\boldsymbol{A x}+\boldsymbol{f}_{\boldsymbol{x}}(\boldsymbol{x}, \boldsymbol{y})=0, \quad \boldsymbol{A} \boldsymbol{y}+\boldsymbol{f}_{\boldsymbol{y}}(\boldsymbol{x}, \boldsymbol{y})=0,
$$

where $A$ is a pentadiagonal banded matrix that depends only on the values of $\alpha, \beta$ and the spatial discretization step $\Delta s$. Here

$$
\begin{gathered}
\boldsymbol{f}_{\boldsymbol{x}}(\boldsymbol{x}, \boldsymbol{y})=\left(f_{x}\left(x_{1}, y_{1}\right), f_{x}\left(x_{2}, y_{2}\right), \ldots, f_{x}\left(x_{n}, y_{n}\right)\right)^{\mathrm{T}}, \\
\boldsymbol{f}_{\boldsymbol{y}}(\boldsymbol{x}, \boldsymbol{y})=\left(f_{y}\left(x_{1}, y_{1}\right), f_{y}\left(x_{2}, y_{2}\right), \ldots, f_{y}\left(x_{n}, y_{n}\right)\right)^{\mathrm{T}}, \\
f_{x}(x, y)=\partial E_{\text {ext }}(x, y) / \partial x \\
f_{y}(x, y)=\partial E_{\text {ext }}(x, y) / \partial y \\
\boldsymbol{x}=\left(x_{1}, x_{2}, \ldots, x_{n}\right)^{\mathrm{T}}, \\
\boldsymbol{y}=\left(y_{1}, y_{2}, \ldots, y_{n}\right)^{\mathrm{T}} .
\end{gathered}
$$


Equations (2) can be thought of as force balance equations; they are satisfied when the snake reaches equilibrium. The snake movement is governed by the following equations:

$$
\begin{aligned}
& \boldsymbol{x}_{t}=(\boldsymbol{A}+\Delta t \boldsymbol{I})\left(\boldsymbol{x}_{t-1}-\boldsymbol{f}_{\boldsymbol{x}}\left(\boldsymbol{x}_{t-1}, \boldsymbol{y}_{t-1}\right)\right), \\
& \boldsymbol{y}_{t}=(\boldsymbol{A}+\Delta t \boldsymbol{I})\left(\boldsymbol{y}_{t-1}-\boldsymbol{f}_{\boldsymbol{y}}\left(\boldsymbol{x}_{t-1}, \boldsymbol{y}_{t-1}\right)\right) .
\end{aligned}
$$

The force balance Equations (2), and therefore snake evolution Equations (3), do not depend explicitly on the image energy $E_{\text {ext }}$. This allows to use more general definition of external forces, which is massively used in various extensions of the active contour model. In the original model, external forces have two serious drawbacks. First, they have low capture range of the edges, that results in the necessity of a very good contour initialization; when initialized far from the desired location, the active contour tends to converge to wrong result because often there are many local minima. The second drawback is that traditional active contour can not adequately detect boundary concavities (especially, the salient ones).

As mentioned before, the actual model used in this paper is based on the GVF snake [18]. It is one of the approaches addressing both drawbacks of the traditional model. It redefines image forces in a more complex way. Given an edge map $M(P)$ (for example, $M(P)=|\nabla I(P)|^{2}$ ), GVF force field $\mathbf{f}(x, y)=$ $\left(f_{x}(x, y), f_{y}(x, y)\right)$ is defined to be the field that minimizes the functional

$$
\varepsilon[\mathbf{f}]=\iint\left(\mu\left(\left|\nabla f_{x}\right|^{2}+\left|\nabla f_{y}\right|^{2}\right)+|\nabla M|^{2}\left(|\mathbf{f}-\nabla M|^{2}\right)\right) d x d y,
$$

where $\mu$ is the regularization parameter that should be set according to the image noise level. In this formulation, the force field retains its smoothness in homogeneous regions, so the edge capture range is kept nearly as large as it is possible.

The correction step incorporates an additional force that attracts the contour to an ellipse. This force acts in the direction normal to the contour, and its magnitude at a control point is proportional to the distance between the point and the ellipse, bounded above by a constant.

Also, pressure forces [3] are used in the correction step. The idea is quite simple: each control point is additionally influenced by the pressure force that acts in normal direction either inwards or outwards.

\section{Obtaining the Optic Disk Boundary}

As mentioned in Sect. 2, the source image is morphologically closed, and the result is used to compute the edge map that defines the GVF force field, which is used in both active contour application steps (the main step and the correction step). In order to decrease processing time, the main step is split in two stages. The first one provides a rough result using large control point intervals and weak termination criteria; at the second stage, smaller control point interval is used and termination criteria are stronger. 
When external force is applied to a control point, it is projected onto the normal to the contour at that point. This prevents movement of control points along the contour, so the difference between model states at different iterations can be expressed in terms of distances between single control point positions at these iterations. We use an inner iteration cycle (which typically consists of $t_{\max }=25$ iterations). Inside this cycle contour points are not redistributed, and a history of contour evolution is stored. Let $\boldsymbol{v}_{i, t}(1 \leqslant i \leqslant n)$ be the $i$-th control point of the contour at $t$-th iteration of the inner cycle, then the termination criteria are:

$$
\left\{\begin{array}{l}
\max _{t \in T} \max _{i: 1 \leqslant i \leqslant n} u_{i, t}<c_{1} \\
\max _{t \in T} \frac{1}{n} \sum_{i=1}^{n} u_{i, t}<c_{2}
\end{array}\right.
$$

where $u_{i, t}=\left|\boldsymbol{v}_{i, t}-\boldsymbol{v}_{i, t_{\max }}\right|^{2}, T=\left\{t_{1}, t_{1}+1, \ldots, t_{\max }-1\right\}, c_{1}$ and $c_{2}$ are constants that define the required accuracy depending on the step being carried out, and $t_{1}=4$ is used to discard the effect of contour relaxation after its redistribution, which may cause significant changes to control point positions.

The correction step incorporates additional ellipse fitting and pressure forces. The intermediate contour is approximated by an ellipse using a fitting procedure that minimizes the sum of the squares of the distances, where each control point $\boldsymbol{v}_{i}$ have its weight equal to $1-D\left(\boldsymbol{v}_{i}\right)$. The maximal ellipse fitting force magnitude is set to a relatively low value so that this force does not supersede image forces in the presence of a strong edge.

The pressure force slightly pushes the contour outwards in the regions where it is crossed by blood vessels. The magnitude of pressure force for $k$-th control point and the smoothness coefficient $\beta_{k}$ depend on the corresponding values in vessel map $D$. Let

$$
d_{k}=\frac{1}{\Delta s} \int_{(k-1) \Delta s}^{(k+1) \Delta s}\left(1-\frac{|s-k \Delta s|}{\Delta s}\right) \cdot D(\boldsymbol{v}(s)) d s
$$

then $\beta_{k}=\left(1-d_{k}\right) \beta_{\text {normal }}+d_{k} \beta_{\text {vessel }}, \beta_{\text {normal }}$ is equal to the value used in the second stage of the previous step, $\beta_{\text {vessel }}$ is the value meant to impose very strong smoothness constraint, and $p_{k}^{*}=P_{\max }\left(1-d_{k}\right)$, where $p_{k}^{*}$ is a preliminary value for the pressure force magnitude, and $P_{\max }=0.15$ is a constant denoting maximal pressure force value.

We also define two additional constants: threshold $P_{\mathrm{thr}}=0.1$ and a lowered pressure force value $P_{\text {low }}=P_{\max } / 2$. The preliminary values vector $\mathbf{p}^{*}$ is convolved with Gaussian $G_{\sigma}$, where value of $\sigma$ is selected so that the corresponding contour part length (assuming that control points are distributed uniformly) is equal to 10 pixels. Let $\tilde{\mathbf{p}}^{*}=\mathbf{p}^{*} * G_{\sigma}$ (note that $\mathbf{p}^{*}$ must be treated as discrete periodic function), then final pressure force magnitude values $p_{k}$ are selected as follows:

$$
p_{k}= \begin{cases}p_{k}^{*} & \text { if } \tilde{p}_{k}^{*} \leqslant P_{\mathrm{thr}} \\ \min \left(p_{k}^{*}, P_{\text {low }}\right) & \text { if } \tilde{p}_{k}^{*}>P_{\mathrm{thr}}\end{cases}
$$


The aim of this procedure is to minimize the chance of excessive inflation of the contour. When there are areas with very dense blood vessels, the pressure is applied to a large number of adjacent contour points, making it possible for the contour to move away from the optic disk boundary. Using a lowered pressure force in such areas restores the balance.

The active contour receives new $\beta_{k}$ values and is evaluated with pressure forces applied to it. In most cases this step requires very few iterations. When the contour reaches equilibrium, it becomes the final result.

\section{$5 \quad$ Results and Concluding Remarks}

We tested our method on retinal images from two databases: MESSIDOR [12], kindly provided by the Messidor program partners, and DRIVE [15]. An expert ophthalmologist provided us with ground-truth optic disk contours for DRIVE images. Hand OD segmentations for MESSIDOR images, which we used in our testing, are currently available at [5]. Sample results of different quality are shown in Fig. 2; the measured accuracy of these results is also given.

We adopt a natural method of measuring the result accuracy $A$, the overlapping of the result $R$ and the ground-truth contour $G: A=S(R \cap G) / S(R \cup G)$, where $S$ denotes area. The overlapping degree is also used in [1], [8]. Table 1 shows percentage of images for several accuracy intervals, obtained by the compared methods, along with the mean accuracy of each method. In [1] the results of testing on MESSIDOR images are presented in the same manner and are included in Table 1. We did not include the method of Lalonde et al. [8] in the comparison, since Aquino et al. [1] obtained significantly better results. Table 1 also contains results of the proposed method for DRIVE images and results of the simple method based on GVF snake with color morphology preprocessing for both databases.

Table 1. Accuracy comparison using the contour overlapping. $M$ and $D$ denote MESSIDOR and DRIVE databases respectively.

\begin{tabular}{lcccccccc}
\hline Method & DB & $A \geqslant 0.95$ & $A \geqslant 0.9$ & $A \geqslant 0.85$ & $A \geqslant 0.8$ & $A \geqslant 0.75$ & $A \geqslant 0.7$ & $\bar{A}$ \\
\hline$[1]$ & $\mathrm{M}$ & $7 \%$ & $46 \%$ & $73 \%$ & $84 \%$ & $90 \%$ & $93 \%$ & 0.86 \\
\hline GVF & $\mathrm{M}$ & $10.7 \%$ & $39.3 \%$ & $52.4 \%$ & $59.2 \%$ & $66.8 \%$ & $72.7 \%$ & 0.77 \\
\hline Proposed & $\mathrm{M}$ & $20.9 \%$ & $68.4 \%$ & $82.6 \%$ & $88.6 \%$ & $91.7 \%$ & $93.7 \%$ & 0.89 \\
\hline GVF & $\mathrm{D}$ & $17.5 \%$ & $40.0 \%$ & $45.0 \%$ & $50.0 \%$ & $57.5 \%$ & $62.5 \%$ & 0.74 \\
\hline Proposed & $\mathrm{D}$ & $30.0 \%$ & $77.5 \%$ & $77.5 \%$ & $80.0 \%$ & $82.5 \%$ & $92.5 \%$ & 0.89 \\
\hline
\end{tabular}

Aquino et al. claim that their method produces much better results (compared to the deformable model [10]) in the cases where an excellent segmentation is impossible to obtain due to poor contrast and/or severe pathologies. In a comparison where segmentation results were divided into excellent, good, fair and 

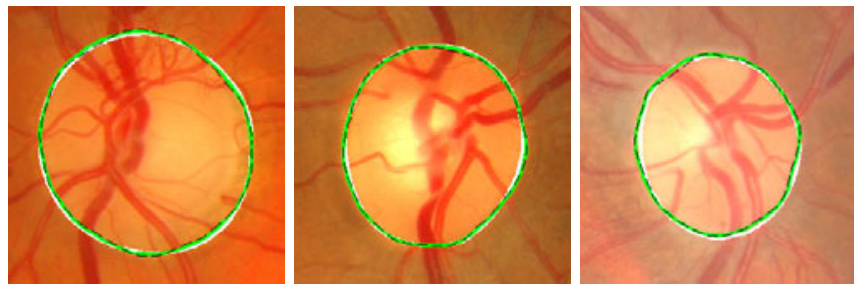

Excellent (left: 0.971, center: 0.979, right: 0.956)
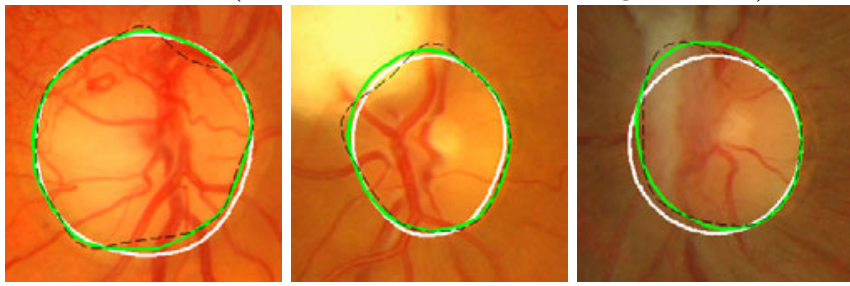

Good/medium (left: 0.942, center: 0.916, right: 0.86)
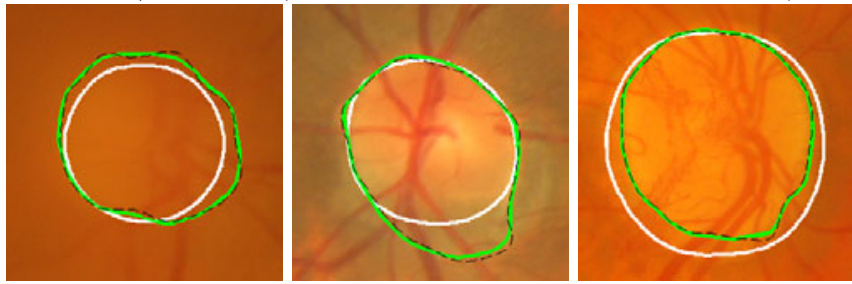

Bad (left: 0.795, center: 0.817, right: 0.804)

Fig. 2. Examples of segmentation results of different accuracy obtained with our method. Ground truth is shown in white, method result is shown in green, and the black dashed line represents intermediate contour before the correction stage.

poor, their method was inferior to the method of Lowell et al. only in the percentage of excellent segmentations ( $40 \%$ versus $42 \%$ ), while the percentage of good and fair results was significantly higher (39\% vs. $31 \%$ and $18 \%$ vs. $10 \%$ respectively). Comparing the results of testing on the MESSIDOR database, Table 1 shows that the proposed method has higher rate of good and fair segmentations, as well as excellent ones, than [1]. The comparison between [1] and [10] gives us an opportunity to compare our method with the Lowell's (though not strictly because of different accuracy measures). It seems that their excellent segmentation quality roughly corresponds to overlapping greater than about 0.91 . It is mentioned that they obtained excellent results in $42 \%$ of the cases, while our method achieves overlapping greater than 0.91 in $63.2 \%$ cases. It seems improbable that their method has a higher rate of very precise segmentations $(A \geqslant 0.95)$, and it is clear that in more difficult cases our method performs much better.

OD segmentation accuracy is also often measured in terms of binary classification of the image pixels: true positive $T_{p}$, true negative $T_{n}$, false positive $F_{p}$ and false negative $F_{n}$ pixel numbers are counted, and then the accuracy is expressed in terms of true positive rate (sensitivity) $R_{T P}=T_{p} /\left(T_{p}+F_{n}\right)$, true negative rate (specificity) $R_{T N}=T_{n} /\left(T_{n}+F_{p}\right)$, positive predictive value, 
or precision rate, which is defined as $P=T_{p} /\left(T_{p}+F_{p}\right)$, and $F$-measure $F=$ $2 \cdot R_{T P} \cdot P /\left(R_{T P}+P\right)$. These values suit well in case of binary classification of independently treated objects, but are not very adequate when used to measure similarity between two contours. Note that contour overlapping, which can be expressed as $T_{p} /\left(T_{p}+F_{p}+F_{n}\right)$, is always not greater than $R_{T P}$ and $P$, and $R_{T N}$ does depend on the size of the image part that is used for OD segmentation: counting pixels of the entire image instead of a cropped part of it would result in very high specificity values, and these values will have little meaning.

We calculated these characteristics for our method and got the following average values: $R_{T P}=94.1 \%, R_{T N}=98.4 \%, P=94.3 \%, F=0.94$. The implicit active contours-based method in [14] achieved $90.67 \%$ average sensitivity and $94.06 \%$ average specificity; a local database of 148 images was used for testing. Duanggate et al. [4] reported average $R_{T P}$ and $P$ values of $85.37 \%$ and $82.87 \%$ respectively on cropped images. It is noted that the testing was done using two datasets: one containing mostly images with clearly visible OD (123 images total), and the second where ODs were mostly faint and unclear (91 image). In [6], the method was tested on 138 images, resulting in the following average values: $R_{T P}=96 \%, P=98 \%$ and $F=0.97$.

Apparently, the proposed method provides better segmentations than [14] and [4]. The method presented in [6] possibly performs better, but in order to make a strong conclusion a rigorous testing of the two methods is needed involving the same retinal image database, ground truth and accuracy metrics. Unfortunately, Joshi et al. did not provide the results of testing their method on publicly available retinal image databases. It is noted that the method was tested on high quality OD-centric images, while DRIVE and MESSIDOR databases contain a noticeable amount of images where it is hard to achieve good OD segmentation, i.e. blurred and/or low-contrast images, or images with the OD located near the edge of camera field of view and affected by edge flare.

Thus testing shows that the method presented in this paper outperforms many other OD segmentation methods and has a high rate of very good segmentations. A great increase in accuracy, comparing to a simple GVF snake algorithm with morphological preprocessing, is achieved by using the blood vessel map information in all processing steps.

Acknowledgments. The work was supported by the federal target program "Scientific and scientific-pedagogical personnel of innovative Russia in 20092013" and a Moscow government grant.

\section{References}

1. Aquino, A., Gegundez-Arias, M., Marin, D.: Detecting the Optic Disc Boundary in Digital Fundus Images Using Morphological, Edge Detection and Feature Extraction Techniques. IEEE Transactions on Medical Imaging 29, 1860-1869 (2010)

2. Chan, T., Vese, L.: Active contours without edges. IEEE Trans. Image Processing 10(2), 266-277 (2001) 
3. Cohen, L.: On active contour models and balloons. CVGIP: Image Understanding 53(2), 211-218 (1991)

4. Duanggate, C., Uyyanonvara, B., Makhanov, S.S., Barman, S., Williamson, T.: Parameter-free optic disc detection. Computerized Medical Imaging and Graphics 35(1), 51-63 (2011)

5. Expert system for early automated detection of DR by analysis of digital retinal images project website. Univ. Huelva, Huelva, http://www.uhu.es/retinopathy

6. Joshi, G.D., Gautam, R., Sivaswamy, J., Krishnadas, S.R.: Robust optic disk segmentation from colour retinal images. In: Proceedings of the 7th Indian Conference on Computer Vision, Graphics and Image Processing, pp. 330-336 (2010)

7. Kass, M., Witkin, A., Terzopoulos, D.: Snakes: Active contour models. International Journal of Computer Vision 1(4), 321-331 (1988)

8. Lalonde, M., Beaulieu, M., Gagnon, L.: Fast and robust optic disc detection using pyramidal decomposition and Hausdorff-based template matching. IEEE Trans. Med. Imaging 20(11), 1193-1200 (2001)

9. Li, H., Chutatape, O.: Boundary detection of optic disk by a modified ASM method. Pattern Recognition 36(9), 2093-2104 (2003)

10. Lowell, J., Hunter, A., Steel, D., Basu, A., Ryder, R., Fletcher, E., Kennedy, L.: Optic nerve head segmentation. IEEE Trans. Medical Imaging 23(2), 256-264 (2004)

11. Mendels, F., Heneghan, C., Thiran, J.: Identification of the optic disk boundary in retinal images using active contours. In: Proc. Irish Machine Vision and Image Processing Conference on Cerebrovascular Diseases, pp. 103-115. IEEE, Los Alamitos (1999)

12. MESSIDOR: Digital Retinal Images, MESSIDOR TECHNO-VISION Project, France, http://messidor.crihan.fr/download-en.php

13. Osareh, A., Mirmehdi, M., Thomas, B., Markham, R.: Colour morphology and snakes for optic disc localisation. In: 6th MIUA Conference, pp. 21-24 (2002)

14. Siddalingaswamy, P., Gopalakrishna, P.: Automatic Localization and Boundary Detection of Optic Disc Using Implicit Active Contours. International Journal of Computer Applications 1(6), 1-5 (2010)

15. Staal, J., Abramoff, M., Niemeijer, M., Viergever, M., van Ginneken, B.: Ridgebased vessel segmentation in color images of the retina. IEEE Trans. Medical Imaging 23(4), 501-509 (2004)

16. Tang, Y., Li, X., von Freyberg, A., Goch, G.: Automatic segmentation of the papilla in a fundus image based on the $\mathrm{C}-\mathrm{V}$ model and a shape restraint. In: Proc. ICPR, pp. 183-186 (2006)

17. Winder, R.J., Morrow, P.J., McRitchie, I.N., Bailie, J.R., Hart, P.M.: Algorithms for digital image processing in diabetic retinopathy. Computerized Medical Imaging and Graphics 33(8), 608-622 (2009)

18. Xu, C., Prince, J.: Snakes, shapes, and gradient vector flow. IEEE Trans. Image Processing 7, 359-369 (1998)

19. Xu, J., Chutatape, O., Sung, E., Zheng, C., Chew, P.: Optic disk feature extraction via modified deformable model technique for glaucoma analysis. Pattern Recognition 40(7), 2063-2076 (2007) 